\title{
La epistemología médica de Claude Bernard*
}

Claude Bernard's Medical Epistemology

César Lorenzano ${ }^{\dagger}$

\section{Resumen}

El artículo muestra que el método experimental de la fisiología, que Claude Bernard expone casi setenta años antes que Karl Popper, coincide hasta en sus términos con el método hipotético-deductivo. Sin embargo era y es mayormente desconocido por los filósofos de la ciencia pese a que se estructurando las investigaciones del área biológica, o los escritos psicoanalíticos de Freud -que como buen neurofisiólogo formado en París conocía a fondo-.

La reconstrucción de las investigaciones en las que Claude Bernard descubre los mecanismos que hacen que el nivel de glucosa en sangre sea constante aunque la ingesta de hidratos de carbono no lo sea, el caso inicial, paradigmático, de la fisiología, permite identificar un núcleo específico de términos interrelacionados -una estructura- que hace que la comunidad de fisiólogos investigue valores que permanecen constantes en los más diversos procesos -digestivos, hormonales, neurológicos, circulatorios-y desentrañe los mecanismos -homeostáticos- que lo posibilitan.

Se propone, además que la noción de homeostasis -central para la fisiología- es retomada como retroalimentación en la cibernética y como regulación en la economía.

La filosofía de la medicina, todavía en cuestión en 1976, había señalado el camino del método y la estructura de la ciencia mucho tiempo antes que la filosofía de la ciencia. Pero esta no lo sabía.

Palabras clave: Claude Bernard - hipotético-deductivismo - epistemología médica

\begin{abstract}
The article shows that the experimental method of physiology, which Claude Bernard exposes almost 70 years before Karl Popper, coincides even in his terms with the hypothetical deductive method. This fact was, however, largely unknown to the philosophers of science in spite of it structured the biological research, or Freud's psychoanalytic writings - due to that he knew the method when he stayed in Paris as a neurophysiologist.

The reconstruction of the research in which Claude Barnard discovers the mechanisms that make the blood glucose level constant even though the carbohydrate intake is not, the initial paradigmatic case of physiology, allows us to identify a specific nucleus of interrelated terms - a structure - that makes the community of physiologists investigate values that remain constant in the most diverse processes - digestive, hormonal, neurological, circulatory - and unravel the mechanisms - that make it possible.

It is proposed, furthermore, that the notion of homeostasis - central to physiology - is taken as feedback in cybernetics and as regulation in the economy.

The philosophy of medicine, still at issue in 1976, had pointed to the path of method and structure of science long before philosophy of science. But it did not know it.
\end{abstract}

Keywords: Claude Bernard - hipothetico-deductivism - medical epistemology

\footnotetext{
* Recibido: 11 de octubre de 2015. Aceptado con revisiones: 5 de diciembre de 2015.

+ Universidad Nacional de Tres de Febrero, Argentina. Para contactar al autor, por favor, escribir a: clorenzano@gmail.com. Metatheoria 6(2)(2016): 49-63. ISSN 1853-2322. eISSN 1853-2330.

(c) Editorial de la Universidad Nacional de Tres de Febrero. Publicado en la República Argentina.
} 


\section{Introducción}

En 1976, las Actas del Encuentro Bianual de la Asociación de Filosofía de la Ciencia de Estados Unidos, dedican algunas de sus secciones a cuestiones relativamente nuevas -en ese entonces- como las que plantean la sociobiología, la tecnología, o los sistemas complejos difusos.

En este contexto, el título de una de las secciones es en sí mismo una definición "¿Qué tiene que ver la filosofía de la medicina con la filosofía de la ciencia?" invitando a que sus ponencias discutan la pertinencia de llamar filosofía de la ciencia a las reflexiones metateóricas sobre la medicina.

Cuatro especialistas (Wartofsky, Engelhardt, Greene y Whitbeck) responden afirmativamente a la pregunta a través de sus artículos, en los que fijan, también desde el título de una de las ponencias, la de Caroline Whitbeck, "La relevancia de la filosofía de la medicina para la filosofía de la ciencia". La bibliografía que esgrimen es aleccionadora de la escasa producción filosófica sobre el conocimiento médico. Aparecen pocos nombres, entre ellos los de George Canguilhem, Stephen Toulmin, o Tristan Engelhardt, quien escribe uno de los artículos (Canguilhem 1963, 1965, 1966, Toulmin 1976, Engelhardt \& Spicker 1974).

Lo menos que podemos acotar es que la filosofía de la ciencia se interesó por la medicina demasiado tardíamente. Como si se desconociera que hace más de un siglo esta disciplina había adquirido la estructura teórica que hoy la caracteriza, avanzando desde entonces a pasos agigantados en el conocimiento de ese microcosmos particular que es el cuerpo humano en salud y enfermedad, con enormes consecuencias para la ciencia y la vida cotidiana. Como si se obviara que su comunidad científica es una de las más numerosas, y que sus representantes más prominentes reciben año tras año el premio Nobel.

¿Por qué sucede esto? Podemos adelantar algunas respuestas. Para algunos, la medicina consiste en la práctica cotidiana de los médicos, sin que se tome en cuenta al conocimiento médico -que incluye ciencias básicas como la fisiología, o la clínica, con su amplia constelación de disciplinas presupuestas-, expuesto en libros de texto y artículos de investigación.

Para otros, su análisis es innecesario, puesto que no es más que biología, aunque no se puedan reducir sus enunciados específicos a los de esa ciencia, ni deducirlos de ella, entre otros motivos porque responden a un campo problemático específico que surge de la salud y la enfermedad humana, a las que son ajenas las teorías biológicas, que hablan de las células, la genética o la evolución de las especies.

Acostumbrados a pensar que la ciencia por antonomasia es la física, se suele identificar la filosofía de la ciencia con las reflexiones que la toman como objeto. El precio que se paga es el desconocimiento de otras disciplinas, y el empobrecimiento del horizonte cognitivo y epistemológico, roto recién en los últimos años por el estudio de las teorías de la biología.

En este artículo me propongo mostrar no sólo la pertinencia del conocimiento médico para la filosofía de la ciencia, sino también la importancia epistemológica de las reflexiones originadas en el interior del campo médico.

Tomaré para ello la obra científica y filosófica de Claude Bernard para exponer la originalidad de su pensamiento metodológico -que anticipa en aproximadamente setenta años el de Karl Popper-, y de su concepción de ciencia, enraizada en su propia disciplina, la fisiología, a la que funda. Mostraré además cómo el método propio de la fisiología es impensable sin la existencia de un campo teórico médico, como respuesta al problema que las enfermedades suscitan a las sociedades humanas.

Cuando lo haya hecho, se concordará conmigo que se encuentra en este autor una metodología general explícitamente expuesta como tal; una teoría de la medicina que se construye al compás de la fisiología, y una metodología especial de la fisiología, constituyendo una contribución desde el campo científico y epistemológico de la medicina que la filosofía de la ciencia general no puede descuidar. 


\section{El hipotético-deductivismo de Claude Bernard}

Presentar a Claude Bernard como hipotético-deductivista implica romper con un triple prejuicio.

El primero de ellos -común entre filósofos inadvertidos- es suponer que su opus máximo, Introducción al estudio de la medicina experimental, sólo se refiere a técnicas de laboratorio. Si superaran el espinoso título, encontrarían en él un refinado pensamiento epistemológico (Bernard 1865).

El segundo consiste en pensar que su contribución en este terreno es quizás elemental y sin mayor actualidad. Se desconoce el hecho de que existen ediciones contemporáneas de sus escritos, leídos por todo aquel que se inicia en la investigación fisiológica o clínica, tanto en Francia como en otros países de tradición latina, y que son recomendados durante generaciones por Premios Nobel de fisiología a sus discípulos.

El tercero, es situar su pensamiento metodológico dentro del positivismo de la época. Seguramente quienes lo sitúan dentro de esta corriente filosófica, no tomaron en serio sus afirmaciones antipositivistas, caracterizando a los que pertenecen a esta corriente epistemológica como aquellos que hablan de ciencia sin practicarla. Tampoco consideraron sus afirmaciones antiinductivistas, como cuando expresa: "cuando pensamos que vamos de un hecho especial a un principio, a saber, haciendo una inducción, realmente estamos haciendo una deducción", para terminar diciendo: "resumiré diciendo que a mí me parece que sólo hay una forma de razonamiento, la deducción mediante el silogismo" (Bernard 1959, pp. 76-77). Una forma tan tajante de rechazar la inducción sólo encuentra parangón en Karl Popper, cuando dice: "Ahora bien, en mi opinión, no existe nada que pueda llamarse inducción” (Popper 1973, p. 39).

Introduje -deliberadamente- citas paralelas de Claude Bernard y de Karl Popper con respecto a la inducción. Uno de los hechos más notables de la historia de la epistemología, es el de que Claude Bernard expuso la metodología hipotético-deductivista setenta años antes que Popper, y empleando además de argumentos similares- casi las mismas palabras. Es asimismo notable que apenas se haya señalado esta circunstancia, debido quizás a que no sea un texto de lectura habitual para la comunidad epistemológica. Incluso cuando Peter Medawar -quien no casualmente es un destacado investigador en el área de la inmunología, al punto de obtener el premio Nobel en 1960 por sus indagaciones en este ámbito- historia los antecedentes del pensamiento de Karl Popper en un artículo realizado en homenaje suyo, únicamente menciona a través de dos citas y de apenas media página el uso de las hipótesis como un elemento central de la filosofía de la ciencia de Claude Bernard (Medawar 1974). Ante la mención de este autor, y la de otros -tal como William Whewell-, la respuesta de Popper es que no los había leído, y pensaba que su camino comenzaba con una ruptura con sus antecesores (Popper 1974).

A fin de remarcar estas coincidencias, adoptaré la estrategia de presentar la secuencia estándar de pasos del método hipotético-deductivo según Karl Popper, mencionando a continuación el pensamiento de Claude Bernard en cada uno de ellos. Para los que quieran comparar sus lenguajes, citaré a pie de página la correspondiente enunciación de Popper.

\subsection{El inicio del método, el problema}

Claude Bernard es consciente de que el inicio de la investigación, el paso inicial que dispara el método hipotético-deductivo, es una incógnita, un problema que tiene su origen tanto en la observación, como en la teoría. En esto, su propuesta es más clara que la de Popper, quien tiende a pensar más en términos de problemas empíricos, que teóricos. 


\subsection{La invención de las hipótesis: la intuición}

Sabemos que una vez identificado un problema, para el método hipotético-deductivo no existe una lógica del descubrimiento, ni un método para llegar a las hipótesis con las que se intenta solucionarlo. Ese proceso por el cual se sugieren respuestas, se lo llama intuición. ${ }^{1}$

Expresa Claude Bernard con respecto a una lógica o método para inventar hipótesis: "A propósito de una observación dada, no pueden darse reglas fijas para hacer nacer en el cerebro una idea exacta y fértil. El método experimental no puede dar ideas nuevas y provechosas a los hombres que carecen de ellas", para agregar a continuación: "El sentimiento o la intuición da lugar a la idea experimental o hipótesis” (Bernard 1959, pp. 60-62).

Así como remarca anteriormente el papel de la teoría para visualizar un problema relevante además del rol jugado por la experiencia-, ahora insiste en que es la experiencia la que tiene mucho que decir a la hora de formular una hipótesis, al contrario de Popper, para el cual el proceso intuitivo es mucho más abierto, sin asignarle a lo empírico una importancia mayor que al mito, la metafísica, o los sueños. Cuando Claude Bernard dice: "La idea experimental no es en absoluto arbitraria, o puramente imaginativa: tiene que apoyarse en la realidad observada, es decir, en la naturaleza”, no hace más que traducir en palabras las características de su método experimental, para el cual es central la experiencia exploradora, aquella que se realiza sin demasiados preconceptos, sólo para ver qué ocurre, pero a partir de la cual se formula una hipótesis. Falta muy poco para que llame a este procedimiento abducción, como llama Peirce a la inferencia que lleva de los hechos y la teoría a una hipótesis plausible que explique los fenómenos (Peirce 1978), y que Hanson retoma más recientemente (Hanson 1977).

\subsection{El método: hipótesis, deducción, contrastación}

Para el método hipotético-deductivo, las hipótesis se ponen a prueba deduciendo a partir de ellas consecuencias observacionales, cuya verdad o falsedad dice sobre la verdad o falsedad de las hipótesis de las que se parte.

El fuerte papel que juega el concepto de hipótesis en el sistema metodológico de Claude Bernard en momentos en que todavía pesa sobre éste el anatema newtoniano de "hipotesis non fingo", queda reflejado en los siguientes párrafos, que contienen, como en el pensamiento de Popper, la impronta de un racionalismo crítico:

La gente que condena el uso de las hipótesis y de las ideas preconcebidas en el método experimental, comete el error de confundir la invención de un experimento con la observación de sus resultados. [...] Es imposible concebir un experimento sin una idea preconcebida. [...] La esencia del razonamiento experimental será siempre una idea que introducimos en un trozo de razonamiento experimental con el fin de someterla al criterio de los hechos, a saber, del experimento. (Bernard 1959, pp. 50, 72)

La esencia del método es que deben plantearse aquellas circunstancias que podrían refutar las hipótesis, pero no lo hacen; sólo de esta manera, poniéndolas a prueba de la manera más rigurosa, es posible seguir manteniéndolas, sin que esto signifique que han sido verificadas. Agreguemos que la refutabilidad es el criterio de cientificidad, el criterio de demarcación entre ciencia y no ciencia que nos propone Popper. ${ }^{2}$

\footnotetext{
${ }^{1}$ Dice Popper: "Sin embargo, mi opinión del asunto -valga lo que valiere- es que no existe, en absoluto, un método lógico de tener nuevas ideas, ni una reconstrucción lógica de este proceso. Puede expresarse mi parecer diciendo que todo descubrimiento contiene «un elemento irracional» o «una intuición creadora» en el sentido de Bergson” (1973, p. 31). Y, señalando el parecido de su opinión con la de Einstein, lo cita: "la «búsqueda de aquellas leyes sumamente universales [...]. No existe una senda lógica -dice - que encamine a estas... Sólo pueden alcanzarse por la intuición, apoyada en algo así como una introyección ('Einfühlung') de los objetos de la experiencia”" (Einstein, A. (1934), Mein Weltbild, Amsterdam: Querido Verlag, p. 168, citado en Popper 1973, pp. 31-32).

2 "El hombre de ciencia, ya sea teórico o experimental, propone enunciados -o sistemas de enunciados- y los contrasta paso a paso. En particular, en el campo de las ciencias empíricas construye hipótesis -o sistemas de teorías- y las contrasta con la experiencia por medio de observaciones y experimentos" (Popper 1973, p. 27). "Pero, ciertamente, sólo admitiré un sistema entre los científicos o empíricos si es susceptible de ser contrastado por la experiencia" (Popper 1973, p. 40). Agregando más adelante: "voy a proponer [...] que se caracterice el método empírico de tal forma que excluya precisamente aquellas vías de eludir la falsación que mi imaginario crítico señala como
} 
La coincidencia de Bernard con estos postulados es completa:

El objeto del método experimental es transformar este concepto a priori (o hipótesis) en una interpretación a posteriori, fundada en el estudio experimental de los fenómenos. El metafísico, el escolástico y el experimentador, todos trabajan con una idea a priori. La diferencia está en que el escolástico impone su idea como una verdad absoluta que él ha hallado, y de la cual deduce entonces consecuencias por lógica solamente. El experimentador, más modesto, por lo contrario, formula una idea como interrogación, como interpretación, como una anticipación más o menos probable de la naturaleza, de la cual deduce lógicamente consecuencias que, de momento a momento, confronta con la realidad mediante el experimento. (Bernard 1959, p. 55)

Aquí tenemos esbozados algunos de los elementos propios de la concepción popperiana de la ciencia que mencionáramos: la hipótesis, la necesidad de deducir de ella, la demarcación de la ciencia con respecto a la metafísica o la escolástica que hace la experiencia. La experimentación es imprescindible, pues como lo señala Claude Bernard: "Las deducciones, aun completamente lógicas, son siempre dudosas, y por lo tanto tiene que recurrirse necesariamente al experimento para verificar las conclusiones del razonamiento deductivo" (Bernard 1959, p. 55). La corrección de la deducción no garantiza la verdad de las conclusiones, y si estas son erróneas, las premisas también lo son.

En cuando a la manera de ponerla a prueba, la coincidencia con Popper es total:

El resultado de esto es que cuando exponemos una idea o un teoría en la ciencia, nuestro objeto no puede ser el conservarla tratando de buscar todo cuanto pueda ir en su apoyo y apartando todo cuanto la debilita. Por el contrario, debemos examinar con el mayor cuidado los hechos que aparentemente podrían acabar con ella, porque el progreso real siempre consiste en cambiar una teoría vieja que comprende pocos hechos, por una nueva que comprende más. (Bernard 1959, p. 69)

Haremos notar que en este párrafo expone asimismo un criterio de progreso en ciencia que tiende a coincidir con el de las corrientes actuales de la filosofía de la ciencia.

Popper plantea provocativamente que las hipótesis son refutables, mas no verificables. Esto es así, pues existe una asimetría de las mismas con respecto a la verificación y la refutación, y que consiste en que si las consecuencias observacionales son verdaderas, no es posible decir que las hipótesis lo son, pues la verdad de las conclusiones no garantiza la verdad de las premisas, mientras que si son falsas, las premisas necesariamente lo son, puesto que si la deducción es correcta, no nos pueden conducir de la verdad a la falsedad.

Claude Bernard lo sabe, y sabe también que todo conocimiento humano es falible, expresándolo en un momento en que se supone que la teoría de Newton es indiscutiblemente verdadera. Sus palabras son las siguientes:

Todas las teorías que sirven de punto de partida para los físicos, químicos y con más razón aún para los fisiólogos, son ciertas hasta tanto se descubran hechos que no incluyen o que las contradicen. [...] Cuando hallamos un hecho que contradice una teoría interesante, tenemos que aceptar el hecho y abandonar la teoría aún cuando la teoría esté apoyada por grandes hombres y aceptada generalmente. (Bernard 1959, pp. 78 y 202)

No quisiera concluir sin dejar de mencionar que Claude Bernard, al igual que Popper, no fue un refutacionista ingenuo -en el sentido de que la experiencia obligue a abandonar una hipótesis a la primera contradicción-, sino que admite que el científico persista en ella, aduciendo ad-hoc contra las evidencias, hasta que las experiencias permitan hacerla triunfar, o finalmente, integrar el hecho refutatorio en una teoría explicatoria alternativa, ya que según él, "los hechos negativos -refutatorios, agregamos nosotros-, cuando se consideran aisladamente, nunca nos enseñan nada” (Bernard 1959, p. 213; lo que figura en cursivas fue añadido por el autor), y que "en realidad, tiene que haber error o insuficiencia en la observación, porque el aceptar un hecho sin causa, es decir, indeterminado en sus condiciones necesarias, es ni más ni menos que la negación de la ciencia” (Bernard 1959, p. 83). 
No es necesario abundar demasiado, ni recordar las experiencias en las que Claude Bernard continúa la investigación atribuyéndole el error no a la hipótesis que pone a prueba, sino a las circunstancias que rodean al diseño experimental -condiciones anatómicas de los animales de experimentación, pureza de las drogas u otros factores no tenidos en cuenta-, poniendo a prueba estas hipótesis ad-hoc hasta encontrar la falla, a fin de corregirla. O si esto no sucede, integrar el hecho refutatorio en una hipótesis alternativa, con lo cual la experiencia se constituye en crucial -en la terminología epistemológica actual-, ya que permite decidir entre una u otra.

La demora en la refutación, desdeñando en primera instancia el hecho refutatorio si la hipótesis es altamente plausible, es acompañada por la convicción de que los hechos no tienen importancia si no son acompañados por una hipótesis o punto de vista que los ordene conceptualmente. Sabemos de la firme posición de Popper al respecto, que concuerda con su posición anti-inductivista y anti-empirista. Claude Bernard manifiesta que:

La idea anticipada o hipótesis, es entonces, el punto de partida necesario de todo razonamiento experimental. Sin ella, no podríamos hacer ninguna investigación ni saber nada; sólo podríamos ir amontonando observaciones estériles. Si experimentamos sin una idea preconcebida, nos moveríamos al azar. (Bernard 1959, p. 61)

Con lo aquí expuesto, creemos haber justificado nuestra afirmación de que, pese al olvido de la filosofía de la ciencia oficial, es Claude Bernard quien introduce los conceptos -e incluso las palabrasque caracterizan al método hipotético-deductivo, desde las investigaciones teóricas, experimentales y metodológicas que acompañan a la fundación de la fisiología, y con ella de la medicina contemporánea, sentando su independencia epistémica como ciencia de la vida.

\section{La fisiología, teoría de la vida}

Cuando en nuestros días tenemos -todavía- que justificar la independencia epistémica de las ciencias médicas, tendemos a olvidar que continuamos una discusión que se inicia hace más de cien años, cuando Claude Bernard funda la fisiología -y con ella el conocimiento médico moderno-, en el curso de sus investigaciones acerca de la producción del azúcar en los organismos vivos. Mientras lo hace, propone una teoría de que rompe con los moldes mecanicistas cartesianos de la época, y defiende la especificidad del método experimental en fisiología.

Es necesario recordar que en esos tiempos se suponía -en consonancia con el pensamiento cartesiano- que la función de los órganos se deduce de su forma anatómica, y que la anatomía comparada permite llegar a "conclusiones verosímiles sobre las características y el uso de cada órgano", puesto que como "la naturaleza presenta en toda clase de animales todas las combinaciones posibles de órganos", podrían observarse entonces los efectos que provoca la presencia o la ausencia de un órgano determinado. Supuestamente, esto hace innecesaria la experimentación en los organismos vivos, que por otra parte se piensa es una estrategia errónea de investigación, ya que su implementación alterar irremediablemente la "máquina" del organismo, falseando los resultados.

Cuando Claude Bernard rechaza estos supuestos, y postula la necesidad de hacer experiencias con seres vivos, separa tajantemente las ciencias de la vida -biología-, de la anatomía y de la química, otorgándole autonomía epistémica y experimental. En sus palabras:

Ni la anatomía ni la química son suficientes para resolver una cuestión fisiológica; es sobre todo la experimentación en los animales que, permitiendo encontrar en un ser vivo el mecanismo de una

\footnotetext{
${ }^{3}$ Si leemos las conocidas palabras de Popper:

Pero si se me ordena «registre lo que experimenta ahora», apenas sé cómo obedecer a esta orden ambigua: ¿he de comunicar que estoy escribiendo?; ique oigo llamar un timbre, vocear a un vendedor de periódicos o el hablar monótono de un altavoz?; ;o he de informar, tal vez, que tales ruidos me llenan de irritación? Incluso si fuera posible obedecer semejante orden, por muy rica que fuese la colección de enunciados que se reuniese de tal modo, jamás vendría a constituirse en una ciencia: toda ciencia necesita un punto de vista y problemas teóricos. (Popper 1973, p. 101)
} 
función, conduce al descubrimiento de fenómenos que ella sola puede iluminar, y que ninguna otra

hacía prever. (Citado en Canguilhem 1968, p. 146)

Hay una cierta circularidad en la manera en que se justifican sus afirmaciones, ya que la certeza de que elige el camino correcto proviene de los resultados de sus experiencias, que a su vez son inseparables de una teoría fisiológica que formula a medida que las realiza. Una profunda coherencia une al método experimental con la teoría que permite, a su vez, planificar las experiencias.

Hacia 1860, la fisiología de Claude Bernard se constituye en la única disciplina biológica -junto con la teoría celular- que congrega el consenso de la comunidad científica a la que pertenecía; ambas demarcan en el mundo moderno el territorio de un nuevo campo de conocimientos, el biológico. Al contrario de sus contemporáneos, Mendel y Darwin, quienes esperaron largos años para ser "redescubiertos" o aceptados, la fisiología que funda persiste hasta el momento, reconociendo en él a su fundador.

Como veremos más adelante, esta ciencia nueva, implica no sólo una metodología, sino también una concepción de la enfermedad y del tratamiento, ligando así el comienzo de las ciencias biológicas a preocupaciones específicamente médicas.

\section{El nacimiento de la fisiología}

Las investigaciones de Claude Bernard se inscriben en el desciframiento de una antigua dolencia, la diabetes, caracterizada en la antigüedad por Arataeus como aquella en la cual "la carne y los miembros se derretían en la orina”, la que poseía un dulzor que Dobson, en el siglo XVIII, demuestra que se debe a la presencia de azúcar.

Cuando Claude Bernard las inicia, se piensa que el azúcar existente en los organismos proviene de la absorción directa de los alimentos, ya que sólo los vegetales la producen, y que la diabetes es un producto -únicamente- de su ingesta inmoderada.

En el curso de sus investigaciones acerca del camino que sigue el azúcar en el organismo, constata luego de dar a un perro una sopa de leche azucarada, y sacrificarlo a continuación, que el azúcar se encuentra en el hígado y en la sangre de las venas -suprahepáticas- que salen de él. Concluye, entonces, que la ruta seguida es desde el intestino al hígado, como una consecuencia natural de la ingesta. Grande fue su sorpresa cuando al repetir la experiencia en un animal al que alimenta sólo con carne, encuentra nuevamente azúcar en el hígado y en la sangre. Comienza entonces a investigar su presencia en cualquier condición de alimentación, incluyendo el ayuno.

Habitualmente, realiza dos mediciones -como forma de control- del contenido de azúcar del hígado recién extraído a un animal de experimentación. En una ocasión -apremiado por el tiempodeja una de ellas sin hacer, posponiéndola para el día siguiente. Contrariamente a lo esperado, el control tardío revela una cantidad de azúcar mayor que la obtenida anteriormente. En una experiencia posterior, lava las venas que salen del hígado "suprahepáticas" -eliminando de ellas toda la glucosa que pudieran contener-, para constatar que al día siguiente había reaparecido. Contra todas las previsiones, las experiencias muestran que el hígado se carga de azúcar después de la muerte, y la vierte a la sangre.

La única interpretación posible de estos hechos es que el azúcar se produce en el hígado, para de allí pasar a la sangre, desde donde se distribuye al resto del organismo para ser consumida como energía y calor. Al profundizar el estudio, encuentra que lo hace a partir de una sustancia amilácea -similar al almidón-, en un proceso en el cual interviene una enzima. La llamó materia glicógeno -glucógeno, "formador de glucosa", en la terminología posterior-. Estos hechos, que comprueban que en los animales el azúcar se forma mediante un mecanismo igual al de los vegetales, refutan la teoría anterior según la cual éstos son los únicos capaces de formar de glucosa.

Las experiencias de Claude Bernard muestran que el hígado es el gran regulador del azúcar en la sangre, puesto que en su ausencia es imposible mantenerla en niveles constantes, y que su extirpación lleva a bajar sus niveles -hipoglucemia- progresivamente, hasta concluir con la muerte; tampoco es 
posible aumentarla por ningún medio, incluso en el caso que al animal de experimentación se le haya provocado previamente una diabetes.

Cuando demuestra que si se pincha el piso del cuarto ventrículo, los animales de experimentación pasan a ser diabéticos, encuentra en el sistema nervioso uno de los factores que inciden sobre el hígado para que éste regule los niveles de azúcar.

Podemos leer hoy estas investigaciones como el inicio de una auténtica revolución científica, en la que se funda la fisiología moderna -y por ende la medicina que conocemos-.

Como acostumbra a suceder en historia de la ciencia, las teorías científicas nuevas se forjan en el curso de investigaciones específicas que construyen lo que es visto retrospectivamente como su primer ejemplo, su ejemplar paradigmático en terminología kuhniana. En el caso de la fisiología, pareciera claro que éste es su modelo fundacional, aquél cuyos patrones sigue toda la investigación fisiológica posterior, y el que fuerza a formular para comprender el fenómeno un conjunto de términos nuevos, que son entonces propios de la teoría fisiológica que nace -sus términos teóricos-, que se encuentran, por otra parte, implícitos en la secuencia de experimentos.

Adelantaremos la hipótesis metacientífica de que el entramado conceptual de la fisiología, tanto en lo que hace a sus términos, como a las relaciones que guardan entre sí -en síntesis, aquello que solemos llamar "teoría"-, son necesarios dados los resultados de los experimentos, y las interpretaciones que hace Claude Bernard de ellos.

El primer concepto al que nos referiremos es al de secreción interna. Aunque no es acuñado por primera vez por Claude Bernard, éste lo toma para insertarlo en su concepción global del funcionamiento de los seres vivos. Se refiere con él al hecho de que el hígado vuelca el azúcar a la sangre -y su extensión natural, todo fenómeno por el cual los distintos órganos vierten sus productos en la sangre-, distinguiéndola de la secreción externa, como la de los jugos pancreáticos.

Como veremos, es solidario de otro término teórico de la fisiología, el de medio interno, por el cual Claude Bernard designa al ambiente líquido en el que viven las células del organismo, y en el que tienen lugar todos sus intercambios vitales. La manera que encuentra para hacer inteligible este concepto es comentar que mientras los microorganismos unicelulares viven en contacto con un medio externo, en los organismos complejos sus células viven en un medio interno, cuya composición es constante, lo que permite una vida libre e independiente de las condiciones ambientales. Por supuesto, este medio interno es en primer lugar la sangre, como lo sugiere la experiencia del azúcar fabricado por el hígado.

El tercer término teórico -de homeostasis, acuñado posteriormente por Cannon, pero presente ya como concepto en C. Bernard-, señala que la composición de este medio interno es constante, en un equilibrio dinámico que se mantiene pese a los continuos cambios en el intercambio con el exterior. Conduce directamente a él, el hecho de que son constantes los valores de azúcar pese a las variaciones en la alimentación.

Este concepto es complejo, y exhibe una de las características más notables de la teoría fisiológica, que la hace sustancialmente distinta a las concepciones mecanicistas de la época, por las cuales los órganos son independientes unos de otros, y poseen una función propia -que surge, habitualmente, de su forma específica-, que es asimismo independiente: el estómago digiere, el corazón impulsa la sangre. La noción de medio interno y su homeostasis unifica órganos y función, permitiendo visualizarlas -e investigarlas- como aquello que contribuye fisiológicamente a la gran finalidad de la vida, que coincide con su mantenimiento, y por ende con la constancia de su medio interno. Esta teoría funcional y dinámica, en la cual ciertas constantes se encuentran en equilibrio por la regulación que hacen en ellas múltiples factores, se constituye en un modelo -en el sentido de ejemplo- de teoría, que afirma -como lo quería Claude Bernard- la independencia epistémica de la medicina. No se trata de una sola constante, ni pocos factores, como sucede en la física, sino de muchas constantes -en permanente investigación y hallazgo-, y de una cantidad indefinida de factores, que también deben hallarse e investigarse. Los interrogantes propios de esta teoría consisten en preguntarse cuáles serán unas y otros, aunque al satisfacerlos se empleen herramientas provenientes de la química o de la física. 
En realidad, como toda teoría, el entramado que forma con sus términos es relativamente vacío empíricamente, y sólo señala los sitios a los que debe apuntar la investigación, los huecos que se deben llenar, guiándola con los ejemplos que suministran sus ejemplares paradigmáticos. Los fisiólogos todavía se encuentran abocados a esta tarea.

Quisiéramos remarcar, asimismo es que no es siguiendo la génesis histórica de sus conceptos característicos como comprendemos el significado que poseen, sino que lo adquieren allí, en sus interconexiones en el seno de una teoría científica, la de la fisiología.

No es este el lugar en el que caracterizaremos a la teoría fisiológica, cuestión que amerita un desarrollo propio. Señalemos únicamente que se debieran seguir los parámetros de la concepción estructuralista de las teorías, haciendo énfasis en la estructura de sus ejemplares, priorizándolos por sobre los modelos matemáticos.

\subsection{Presupuestos}

Concluimos esta breve referencia a la construcción histórica de la teoría fisiológica, refiriéndonos a un pequeño conjunto de presunciones previas, términos y disciplinas presupuestas, sin las cuales no pueden pensarse ni comprenderse esas experiencias fundacionales, ni su cristalización en una teoría independiente.

La primera de ellas es que no hay diferencias ni jerarquías entre los reinos animales y vegetales desde el punto de vista de su funcionamiento fisiológico. Esto facilitó captar inmediatamente el significado de la experiencia por la cual aparece glucosa en el hígado en un animal en ayunas, y las que la continuaron, desconcertantes para sus contemporáneos, puesto que no admitían que los animales sintetizaran azúcares, como lo hacen los vegetales (Bernard 1855-1856, entre otros escritos).

La segunda, es concordar en que la unidad funcional del organismo es la célula, y no los órganos o los tejidos. La teoría celular se constituye en una teoría presupuesta para la fisiología, que piensa que "los fenómenos vitales son el resultado del contacto entre las unidades orgánicas de un cuerpo con el medio fisiológico interno", y "el punto fundamental de toda la medicina experimental" (Bernard 1959, p. 107). Son las células las que viven del medio interno, y las que actúan en su equilibrio; en realidad, cuando un fisiólogo se refiere a que un cierto órgano -el hígado, por ejemplo- regula los niveles de glucosa en sangre, lo que dice es que en las células del hígado se produce el azúcar, y son en ellas en las que actúan los distintos factores que las llevan a aumentar o disminuir su producción. Este presupuesto tiene una gran importancia, pues lleva más adelante a buscar las células que realizan determinada función -como sucede en el curso de las investigaciones sobre los hidratos de carbono, que encuentran en las células de Langherjans del páncreas las que se especializan en producir insulina. Con el tiempo, el supuesto de la relación entre células y funciones conduce a la revolución conceptual que continúa la labor de la fisiología con otras herramientas teóricas, y que consiste en buscar las reacciones bioquimicas por medio de las cuales las células cumplen su función específica. Cuando esto sucede, la teoría bioquímica es la que continúa, desde otra perspectiva, los caminos que mostró la fisiología.

La tercera consiste en predicar un determinismo para los organismos vivos. Esto se traduce en la posibilidad de encontrar leyes generales para sus múltiples, infinitos funcionamientos, y por lo tanto, en sentar las condiciones de su cientificidad.

En su siguiente etapa, las investigaciones fisiológicas que continúan a Claude Bernard, hacen intervenir a un actor esencial del metabolismo de los hidratos de carbono, el páncreas, como el primero de una larga serie de factores de origen glandular.

En ellas se muestra con la mayor nitidez una casi constante de la investigación fisiológica, a saber, su permanente ligazón con el estudio de las enfermedades, de tal manera que su conocimiento implicara simultáneamente el del funcionamiento normal. La existencia de las enfermedades son el motor de esa ciencia básica de la medicina, y uno de los medios para comprobar la efectividad de sus postulados, puesto que al menos en lo que se refiere a las dolencias que se caracterizan por un 
aumento o una disminución de alguna función normal, los mecanismos de su producción coinciden con la metodología de laboratorio para determinar la verdad o falsedad de sus afirmaciones.

Nos referiremos a continuación -brevemente- al método de la fisiología, al que mostraremos íntimamente unido a su objeto de estudio, y su racionalidad, que tiene que ver con esta concordancia entre el método y las alteraciones de lo normal, que concluyen en enfermedad.

\section{El método de la fisiología}

Una vez que ha puesto un punto final a la mayor parte de sus investigaciones, Claude Bernard expone los pasos metodológicos que ha seguido en ellas en su texto acerca de medicina experimental. Ya nos hemos referido a su metodología más general, que lo hace uno de los fundadores de lo que hoy se conoce como método hipotético-deductivo. Comentaremos ahora un aspecto más específico de su metodología, más ligado a la exploración que hace la fisiología de los organismos vivos.

De acuerdo a Claude Bernard, son tres -centralmente- las maniobras experimentales con las que se funda la fisiología. La primera de ellas consiste en la extirpación de un órgano determinado, a los efectos de observar las alteraciones que se producen. Por ejemplo, quitar el hígado y ver que los niveles de azúcar caen hasta desaparecer.

En un segundo paso, se constata si efectivamente ese efecto depende de la extirpación, y no de otros factores, se restituye la función que se atribuye al órgano. Si se piensa que la acción es debida a alguna hormona, suministrarla para revertir la acción. Si esto sucede, es que se está en el camino correcto. Podrían suministrarse -por ejemplo- extracto de páncreas para normalizar los niveles de glucosa previamente altos luego de la extirpación.

Por el tercero, se administra en cantidades excesivas el factor que se supone involucrado, y se observa si no sólo restituye la normalidad, sino también la altera en el sentido previsto. Si se piensa que la insulina baja la concentración de glucosa en sangre, su administración excesiva debiera bajarla excesivamente.

Cuando Claude Bernard pone esto en práctica -y posteriormente lo codifica como método-, pareciera que no hace más que seguir la tradición de Hipócrates de cómo debe explorarse metodológicamente en medicina, a la que amplía y específica a los fines experimentales de la fisiología. Hipócrates dice:

Las enfermedades son el resultado de una amplia variedad de causas, y debemos considerar causas seguras de una afección, todas aquellas cosas cuya presencia es necesaria para que aparezca, y cuya ausencia determina su desaparición. (Citado en Laín Entralgo 1973)

Los supuestos metodológicos de Claude Bernard no sólo registran el antecedente de la propia tradición médica, sino que se vinculan asimismo con otra tradición mucho más próxima en el tiempo, la del positivismo de Comte - no olvidemos que Bernard fue vicepresidente de la Sociedad de Biología, en cuyo manifiesto de fundación figura la estricta fidelidad a las enseñanzas de este filósofo-. No es de extrañar entonces que tuvieran una honda similitud con las reglas de Stuart Mill de las concordancias y las diferencias, que expresan en un lenguaje más moderno los dichos hipocráticos, como surge de la simple comparación. Leemos, en efecto en Mill, acorde con Hipócrates, lo siguiente:

Si un caso en el cual el fenómeno que se investiga se presenta y un caso en el cual no se presenta tienen todas las circunstancias comunes excepto una, presentándose ésta solamente en el primer caso, la circunstancia única en la cual difieren los dos casos es el efecto, o la causa, o una parte indispensable de la causa de dicho fenómeno. (Citado en Copi 1974, p. 430)

cuando habla del método de las concordancias. Habiendo dicho antes, mencionándolo como método de las diferencias:

Si dos o más casos del fenómeno que se investiga tienen solamente una circunstancia en común, la circunstancia en la cual todos los casos concuerdan, es la causa o efecto del fenómeno en cuestión. (Citado en Copi 1974, p. 426) 
De la misma manera, Claude Bernard investiga una función observando que la desaparición de un factor provoca determinado efecto funcional, y su restitución lo invierte.

Agregaremos, sin embargo, algunas diferencias que lo alejan de la simple observación y sistematización de datos, como lo pide el positivismo, y que excede al hecho de que Bernard provoca experimentalmente- el fenómeno que quiere explorar.

En primer lugar, como lo hace notar agudamente en sus escritos -transformándose así de positivista en hipotético-deductivista-, la extirpación y observación son apenas unos primeros pasos a partir de los cuales es necesario hacer hipótesis acerca de cuál o cuáles son los factores involucrados en el fenómeno, para continuar de allí el proceso de búsqueda, de hipótesis en hipótesis. En este contexto, la restitución del órgano -o el animal testigo- ponen a prueba las hipótesis, enfrentándolas a instancias refutadoras que deberán superar.

Sin embargo, resta un paso metodológico que queda sin una explicación satisfactoria dentro de los esquemas metodológicos ya citados, y que hacen pensar en que en Claude Bernard todos ellos tienen su origen no sólo en convicciones metodológicas previas -que existieron-, sino en la lógica misma de la investigación fisiológica, que busca el funcionamiento del organismo, a fin de aprender sobre las enfermedades que lo aquejan.

Me refiero a la introducción de la hiperfunción dentro de la metodología fisiológica, que únicamente puede explicarse por su estrecha ligazón con lo patológico -que carece de importancia para Stuart Mill, quien por otra parte, no posee los intereses médicos que son fundamentales para C. Bernard-.

Pero esto implica introducir en la teoría -que ya consta de una estructura vertebrada por los conceptos de medio interno, secreción interna y homeostasis-, una concepción de enfermedad inseparablemente ligada a la de las funciones fisiológicas normales. Esto es así por los siguientes motivos. Si la función de un órgano consiste en su contribución al mantenimiento de un cierto equilibrio -homeostasis-, tanto su extirpación como su hiperfunción lo apartan de él, produciéndose en ambos casos un estado patológico, sea por déficit o por exceso. Extirpar un órgano significa -en este contexto- conocer parte de su función y al mismo tiempo, conocer la enfermedad causa su fallo. También el provocar la hiperfunción obedece a la necesidad de conocer las enfermedades a las que da origen un órgano determinado, ahora no por disminución de su funcionamiento normal, sino de su exceso.

La restitución de la función -el segundo paso del método-, dice, por otra parte, que ese es el factor efectivamente involucrado en la función perdida, pero también dice acerca de la terapéutica a instaurarse -como el tratamiento de la diabetes con insulina-.

Todo esto significa que no hay forma de explorar el funcionamiento de un órgano sin saber simultáneamente de las enfermedades a las que da origen. Al investigar fisiología, se provocan indefectiblemente enfermedades, conociéndose así tanto origen, como los mecanismos que conducen a su curación. O lo que es otra forma de expresarlo, que es imposible separar la fisiología de la patología y la terapéutica.

De esta manera, el método que emplea Claude Bernard coincide con su teoría.

Agreguemos como dato curioso que Siguen Freud se aparta muy poco de esta concepción cuando construye su obra explorando los rasgos enfermos de sus pacientes. Nos dirá, al igual que Claude Bernard, que sólo así conoceremos los mecanismos psicológicos que subyacen en el funcionamiento psíquico normal, que difieren de los primeros en grado, pero no en esencia. Posiblemente no sea una simple coincidencia, puesto que durante su etapa de investigador fisiológico en París, Freud debió conocer a fondo su obra, que pudo impregnar no sólo a su teoría, sino a su profundo y prolongado interés en la estructura metodológica de la ciencia.

Con todo, el esquema presentado no implica que su aplicación garantice casi automáticamente el logro de resultados relevantes. Cuando se lo aplica, la respuesta del organismo exige una interpretación cuidadosa -de ninguna manera trivial-, y nuevas investigaciones para situarlo correctamente en la red de factores homeostáticos.

Las complejidades surgen de la siguiente situación. Un cierto equilibrio en la sangre -por ejemplo, de la glucosa- no se debe a un único factor, sino a una multitud de ellos, que a su vez se encuentran 
interrelacionados entre sí, de tal manera que un aumento de uno determina un aumento o una disminución de los otros. Siendo esto así, cuando se extirpa un órgano -digamos el páncreas-, la hiperglucemia resultante puede atribuirse a diversas circunstancias. Pudiera ser que falta algo que produce el páncreas, o que los nervios que lo rodean son imprescindibles en el mecanismo compensatorio, o que las sustancias que segrega intervienen indirectamente en el fenómeno, inhibiendo o aumentando la cantidad de otra sustancia segregada por otro u otros órganos. Además, es importante investigar si hay un único factor como causa del fenómeno, o si es provocado por un conjunto de ellos, y cuál es la importancia relativa de cada uno de ellos.

Para resolver cada uno de estos enigmas, que surgen a cada paso de las investigaciones, el tronco central de las experiencias se ramifica en todos estos sentidos -para lo cual es necesario hacer hipótesis acerca de cada uno de estos factores, ya que se los desconoce de entrada-, eliminándolos o corroborándolos, para encontrarse que abren un abanico de nuevas alternativas.

Es necesario eliminar asimismo los errores de laboratorio, la pertinencia de la especie animal elegida para la investigación, etcétera.

Incidentalmente, esto da al método hipotético deductivo de Claude Bernard una característica que lo diferencia notablemente del que expusiera Karl Popper: mientras que para Karl Popper, existen problemas e hipótesis aisladas, siendo azaroso el número de hipótesis que se explorarán, en Claude Bernard las hipótesis son parte de la exploración de factores que se encuentran -en principiolimitados por el universo de la investigación, y que tienen que ver con su concepto de medio interno, y el número limitado -aunque amplio- de variables en juego.

Si nos permitimos una vuelta de tuerca más sobre la primacía de las necesidades teóricas y empíricas de la fisiología como factor determinante en la propuesta metodológica, diremos que la manera en que Claude Bernard expone el método hipotético-deductivo, es enteramente funcional a sus necesidades de investigación, a su vez inseparable de la fisiología.

En él, la formulación de las hipótesis proviene de la teoría, o del experimento para observar; y el universo de hipótesis admisibles se encuentra delimitado por los órganos, las células y las funciones en juego.

Agreguemos que al actuar, como hemos visto, en el seno de una teoría ya establecida, y que por supuesto - de acuerdo a las concepciones más actuales de la evolución de la ciencia, como la de Thomas Kuhn-, se desarrolla respondiendo las incógnitas que deja abiertas, puede ser visto como el método que guía la investigación en el seno de los paradigmas, en el curso de su ciencia normal. En este contexto, la creatividad, la intuición, se reserva para la resolución de los problemas propios de cada teoría, que brinda el marco en el que es lícito formularlos, y el tipo de respuestas que necesitan.

\section{El método de la fisiología}

En nuestro análisis de la obra de Claude Bernard, estudiamos en primer lugar las grandes coincidencias que tiene su metodología general con el hipotético-deductivismo de Karl Popper, y que habitualmente pasa desapercibido. Bernard expone el método experimental de la fisiología casi con los mismos términos con los que lo hace Popper, aunque no utiliza nociones lógicas para fundamentarlo, pero no por ello de manera menos rigurosa y sistemática.

Posteriormente, narramos la sucesión de experiencias acerca de la formación glucosa por el hígado, que se constituye en el ejemplar paradigmático de la naciente teoría fisiológica.

Vimos cómo la experiencia, para ser comprendida adecuadamente -explicada- necesita ser interpretada a la luz de un conjunto de términos interrelacionados, específicos de la fisiología -en suma, términos T-teóricos-.

Algunos de ellos tuvieron una larga influencia, más allá de la fisiología, una teoría que se constituyó en un modelo que siguieron otras disciplinas.

La más notable es la adopción por parte de la cibernética de la noción de homeostasis, aun sin mencionarla, ya que la noción de equilibrio entre factores cuyas alteraciones se compensan 
mutuamente adopta el término de retroalimentación, un punto central de la propuesta de Robert Wiener y Arturo Rosenblueth, los creadores de esta área de conocimientos como disciplina científica. Añadamos que la referencia no es arbitraria, ya que Rosenblueth fue un distinguido cardiólogo y fisiólogo, que realizó con el propio Wiener investigaciones fisiológicas acerca de la conducción de estímulos en las fibras nerviosas (Wiener 1978, Rosenblueth 1981).

Reaparece asimismo en la teoría general de sistemas, así como en la teoría regulacionista (francesa) de la economía (ver Boyer 1989), la que se construye alrededor de un curso de Canguilhem, en el que el concepto central consiste en una paráfrasis de la homeostasis de Claude Bernard.

Si pensamos que sólo las ideas prohíjan ideas -que las estructuras de pensamiento se construyen con estructuras de pensamiento-, entonces hemos mostrado en el transcurso de nuestro escrito, que la filosofía de la ciencia y la ciencia misma, no pueden prescindir del pensamiento médico de Claude Bernard. En realidad, nunca ha prescindido.

La pregunta por una filosofía de la medicina que sea una filosofía de la ciencia especial, formulada en 1976, había sido respondida más de cien años antes.

\section{Síntesis}

En nuestro análisis de la obra de Claude Bernard, estudiamos en primer lugar las grandes coincidencias que tiene su metodología general con el hipotético-deductivismo de Karl Popper, y que habitualmente pasa desapercibido. Bernard expone el método experimental de la fisiología casi con los mismos términos con los que lo hace Popper, aunque no utiliza nociones lógicas para fundamentarlo, pero no por ello de manera menos rigurosa y sistemática.

Posteriormente, narramos la sucesión de experiencias acerca de la formación glucosa por el hígado, que se constituye en el ejemplar paradigmático de la naciente teoría fisiológica.

Vimos cómo la experiencia, para ser comprendida adecuadamente -explicada- necesita ser interpretada a la luz de un conjunto de términos interrelacionados, específicos de la fisiología -en suma, términos T-teóricos-.

Algunos de ellos tuvieron una larga influencia, más allá de la fisiología, una teoría que se constituyó en un modelo que siguieron otras disciplinas.

La más notable es la adopción por parte de la cibernética de la noción de homeostasis, aun sin mencionarla, ya que la noción de equilibrio entre factores cuyas alteraciones se compensan mutuamente adopta el término de retroalimentación, un punto central de la propuesta de Robert Wiener y Arturo Rosenblueth, los creadores de este área de conocimientos como disciplina científica. Añadamos que la referencia no es arbitraria, ya que Rosenblueth fue un distinguido cardiólogo y fisiólogo, que realizó con el propio Wiener investigaciones fisiológicas acerca de la conducción de estímulos en las fibras nerviosas (Wiener 1978, Rosenblueth 1981).

Reaparece asimismo en la teoría general de sistemas, así como en la teoría regulacionista (francesa) de la economía (ver Boyer 1989), la que se construye alrededor de un curso de Canguilhem, en el que el concepto central consiste en una paráfrasis de la homeostasis de Claude Bernard.

Si pensamos que sólo las ideas prohíjan ideas -que las estructuras de pensamiento se construyen con estructuras de pensamiento-, entonces hemos mostrado en el transcurso de nuestro escrito, que la filosofía de la ciencia y la ciencia misma, no pueden prescindir del pensamiento médico de Claude Bernard. En realidad, nunca ha prescindido.

La pregunta por una filosofía de la medicina que sea una filosofía de la ciencia especial, formulada en 1976, había sido respondida más de cien años antes. 
Bibliografía

Bernard, C. (1865), Introduction à l'étude de la médicine expérimentale, Paris: G. Baillière. (Versión castellana: Introducción al estudio de la medicina experimental, Buenos Aires: El Ateneo, 1959.)

Bernard, C. (1855-1856), Leçons de physiologie expérimentale appliquée à la médecine, 2 vols., Paris: G. Baillière.

Bernard, C. (1937), Philosophie (manuscrit inédit), Paris: Hatier-Boivin.

Blainville, H. M. D. de (1833), Cours de physiologie générale et comparée, professé à la Faculté des sciences de Paris, 3 Vols., Paris: G. Baillière.

Boyer, R. (1898), La teoría de la regulación: un análisis crítico, Buenos Aires: Humanitas.

Canguilhem, G. (1963), "La constitution de la physiologie comme science”, en Kayser, C. (ed.), Physiologie, Paris: Flammarion, pp. 11-48.

Canguilhem, G. (1965), La Connaissance de la Vie, Paris: Vrin.

Canguilhem, G. (1966), Le Normal et le Pathologique, Paris: PUF.

Canguilhem, G. (1968), Etudes d'histoire et de philosophie des sciences, Paris: Vrin.

Comte, A. (1830-1842), Cours de Philosophie Positive, 6 vols., Paris: Bachelier.

Copi, I. (1974), Introducción a la lógica, Buenos Aires: EUDEBA.

Engelhardt, H. T. (1977), "Is There a Philosophy of Medicine?", en Suppe, F. y P. D. Asquith (eds.), PSA 1976, Proceedings of the 1976 Biennal Meeting of the Philosophy of Science Association, vol. II, East Lansing, Michigan: Philosophy of Science Association, pp. 94-109.

Engelhardt, H. T. y S. F. Spicker (1974), Evaluation and Explanation in the Biomedical Sciences, Dordrecht: Reidel.

Grene, M. (1977), "Philosophy of Medicine: Prolegomena to a Philosophy of Science”, en Suppe, F. y P. D. Asquith (eds.), PSA 1976, Proceedings of the 1976 Biennal Meeting of the Philosophy of Science Association, vol. II, East Lansing, Michigan: Philosophy of Science Association, pp. 77-94.

Hanson, N. R. (1977), Patrones de descubrimiento. Observación y explicación, Madrid: Alianza Universidad.

Houssay, B. A. (1941), “Claude Bernard y el método experimental”, Revista de la Universidad Nacional de Córdoba 28 (9. 10): $1282-1295$.

Kuhn, T. S. (1971), La estructura de las revoluciones científicas, México: Fondo de Cultura Económica. (Versión castellana de: The Structure of Scientific Revolutions, Chicago: Chicago University Press, 1962, 2a ed. 1970.)

Kuhn, T. S. (1969), "Postscript 1969”, en Kuhn, T. S., The Structure of Scientific Revolutions, Chicago: Chicago University Press, 1970, pp. 174-210. (Versión castellana: "Posdata”, en Kuhn, T. S., La estructura de las revoluciones científicas, México: Fondo de Cultura Económica, 1971, pp. 268-319.)

Laín Entralgo, P. (1973), Historia universal de la medicina, Barcelona: Salvat.

Lorenzano, C. (1977), “Análisis metodológico de una ciencia empírica, la medicina”, Diánoia 23: 124-137.

Lorenzano, C. (1980), “Dos racionalismos críticos: Claude Bernard y Karl Popper”, Teoría, Anuario de filosofía 1(1): 223-245.

Medawar, P. (1974), "Hypothesis and Imagination”, en Schilpp, P. A. (ed.), The Philosophy of Karl Popper, La Salle, IL: Open Court, pp. 274-292.

Peirce, C.S. (1978), Lecciones sobre el pragmatismo, Buenos Aires: Aguilar.

Popper, K. (1973), La lógica de la investigación científica, Madrid: Tecnos.

Popper, K. (1974), "Replies to my Critics, 16. Reply to Medawar on Hypothesis and Imagination”, en Schilpp, P. A. (ed.), The Philosophy of Karl Popper, La Salle, Illinois: Open Court, pp. 1030-1037.

Rosenblueth, A. (1981), Mente y cerebro. Una filosofía de la ciencia, México: Siglo XXI. 
Ruse, M. (1973), La filosofía de la biología, Madrid: Alianza.

Stegmüller, W. (1973), Theorienstructuren und Theoriendynamic, Springer, Heidelberg. (Versión inglesa: The Structure and Dynamics of Theories, New York: Springer, 1976. Versión castellana: Estructura y dinámica de teorías, Barcelona: Ariel, 1983.)

Stegmüller, W. (1979), The Structuralist View of Theories, Berlin: Springer.

Toulmin, S. (1976), “On the Physician's Understanding”, The Journal of Medicine and Philosophy 1: 32-50.

Wartofsky, M. (1977), "How to Begin Again: Medical Therapies for the Philosophy of Science”, en Suppe, F. y P. D. Asquith (eds.), PSA 1976, Proceedings of the 1976 Biennal Meeting of the Philosophy of Science Association, vol. II, East Lansing, Michigan: Philosophy of Science Association, pp. 109-123.

Whitbeck, C. (1977), "The Relevance of Philosophy of Medicine for the Philosophy of Science”, en Suppe, F. y P.D. Asquith (eds.), PSA 1976, Proceedings of the 1976 Biennal Meeting of the Philosophy of Science Association, vol. II, East Lansing, Michigan: Philosophy of Science Association, pp. 123-139.

Wiener, N. (1978), “Mis días en México. 1944”, en Wiener, N., Ensayos científicos, México: CONACYT, pp. $239-254$. 